\title{
Determinanten der Schulleistung
}

Christian Brühwiler • Andreas Helmke

\section{Einführung}

Die Frage nach den Determinanten schulischer Leistungen (engl. causes/correlates/determinants of scholastic achievement) gehört zu den ältesten und zugleich schwierigsten Problemen der Pädagogischen Psychologie. Dies beginnt bereits mit der Definition des Kriteriums: Unter Schulleistung kann sehr Unterschiedliches verstanden werden, z. B. Leistungen individueller Schüler oder von Schulklassen, prozedurales oder deklaratives Wissen, fachspezifisches Wissen oder überfachliche Fähigkeiten ( $\rightarrow$ Metakognition; $\rightarrow$ Lernstrategien und Lernstile; $\rightarrow$ Intelligenz und Begabung). Dazu kommt, dass Leistungen aus unterschiedlicher Perspektive und mit verschiedenen Methoden erfasst werden können: statisch (als Leistung zu einem gegebenen Zeitpunkt) oder dynamisch (als Leistungszuwachs), mit Hilfe von Tests ( $\rightarrow$ Pädagogisch-psychologische Diagnostik; $\rightarrow$ Schultests; $\rightarrow$ Lehrzielorientierte Tests und Lernverlaufsdiagnostik) oder durch Lehrerurteile $(\rightarrow$ Zensuren und verbale Schulleistungsbeurteilung). Die zweite Schwierigkeit liegt in der multiplen Determiniertheit der Schulleistung begründet: Die Schulleistung hängt sowohl von Persönlichkeitsmerkmalen des Lernenden als auch von Familie $(\rightarrow$ Eltern und Familie), Schule, Peers $(\rightarrow$ Soziales Lernen und Peerbeziehungen) und Medien ( $\rightarrow$ Lernen mit elektronischen Medien) ab - und diese verschiedenen Einflüsse stehen in einem komplexen Gefüge zueinander. Die Betrachtung nur eines Ausschnittes oder gar nur einer isolierten Determinante der Schulleistung ist immer mit der Gefahr kurzschlüssiger Interpretationen und Fehlspezifikationen verbunden. In diesem Beitrag kann nur eine knappe Übersicht über Determinanten der Schulleistung gegeben werden; ausführlichere Darstellungen finden sich bei Helmke und Weinert (1997); vgl. auch die Metaanalyse von Wang et al. (1993) sowie die umfassende Studie von Hattie (2009).

\section{Verschiedene Modelle schulischen Lernens}

Determinanten der Schulleistung sind Bestandteil verschiedener Modelle schulischen Lernens, deren Spektrum von der Makroebene wie dem Produktivitätsmodell von Walberg (1990) bis zur Mikroebene wie kognitionspsychologischen Prozessmodellen des Wissenserwerbs (vgl. Steiner, 1996) reicht. Auf einer mittleren Ebene befinden sich die klassischen Modelle des schulischen Lernens (z.B. von Bloom, 1976; Harnischfeger \& Wiley, 1976; Carroll, 1963; vgl. Helmke \& Weinert, 1997; Einsiedler, 1997), bei denen individuelle Lernvoraussetzungen und Merkmale von Schule und Unterricht auf einer relativ allgemeinen Ebene miteinander verknüpft werden. Grundlage vieler empirischer Untersuchungen und Basis für zahlreiche inzwischen vorliegende Metaanalysen sind meist bereichsspezifische Theorien zu einzelnen Determinanten der Schulleistung.

Abbildung 1 gibt eine Übersicht über wichtige Erklärungsblöcke in Gestalt eines Angebots-Nutzungs-Modells. Sie veranschaulicht, dass es neben den individuellen Determinanten vor allem vier Bereiche sind, in denen sich Bedingungsfaktoren der Schulleistung finden lassen: Familie, Schule, Medien und Gleichaltrige. Der Schwerpunkt dieses Artikels liegt auf individuellen und familiären Determinanten (für Informationen zu den anderen Bedingungsfeldern vgl. Helmke \& Weinert, 1997; Hattie, 2009). Auf die Rolle der $\rightarrow$ Unterrichtsqualität wird nur sehr knapp eingegangen.

\section{Individuelle Determinanten}

\section{Kognitive Determinanten}

Neben konstitutionellen Faktoren (biologische Merkmale wie z.B. Lebensalter und Geschlecht sowie die mit bestimmten $\rightarrow$ Lernschwierigkeiten und Lernbehin- 


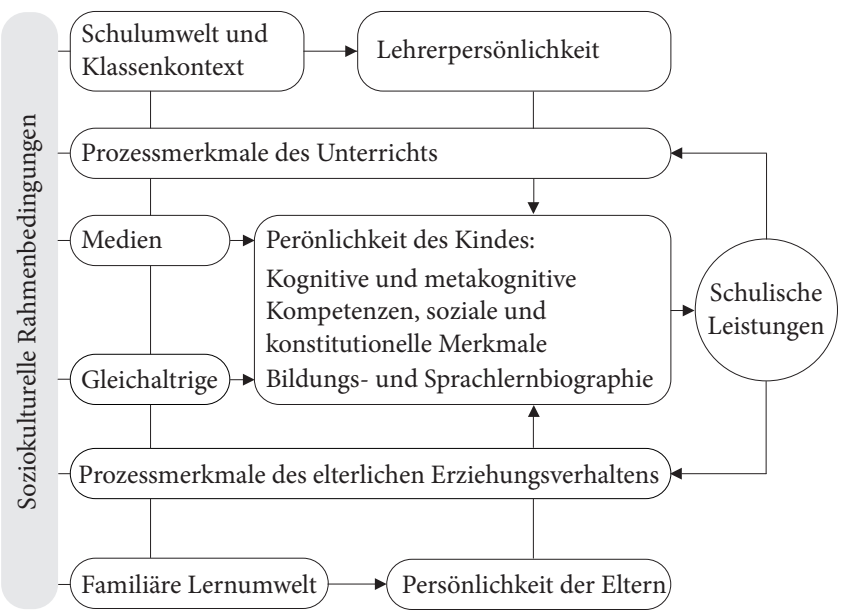

Abbildung 1 Ein Makro-Modell der Bedingungsfaktoren schulischer Leistungen als wesentlich vorhersagestärker erwiesen hat als die allgemeine (also bereichsunspezifische) Intelligenz (vgl. Weinert \& Helmke, 1995a). Dieser Befund ist durch die neuere $\rightarrow$ Expertise-Forschung erhärtet worden (zur Rolle des Vorwissens vgl. Schrader, 2006). Er wird auch durch die Ergebnisse der HattieStudie bestätigt, der zufolge das vorausgehende Leistungsniveau (erfasst durch Leistungstests, aber auch durch Noten) mit einer Effektstärke von $d=0,67 \mathrm{zu}$ den stärksten Prädiktoren der Leistungsentwicklung zählt. derungen verbundenen körperlichen Beeinträchtigungen; vgl. $\rightarrow$ Anlage und Umwelt; $\rightarrow$ Geschlechtsunterschiede) stellt Intelligenz eine der wichtigsten Determinanten der Schulleistung dar $(\rightarrow$ Intelligenz und Begabung). Trotz erheblicher Schwankungsbreite gehört die enge Beziehung zwischen allgemeiner Intelligenz und Schulleistung (mittlere Korrelation von $r=, 50$ bis $r=, 60)$ zu den am besten gesicherten empirischen Befunden (für eine Übersicht vgl. Gustafsson \& Undheim, 1996; Hattie, 2009; Rost, 2013).

Intelligentere Schüler können einerseits schneller und effektiver lösungsrelevante Regeln erkennen und Probleme lösen; andererseits fällt es ihnen leichter, ein besser vernetztes, flexibler nutzbares, »intelligenter « organisiertes Wissen zu erwerben, welches wiederum nachfolgende, darauf aufbauende Lernprozesse begünstigt.

Der enge Zusammenhang zwischen Intelligenz und Schulleistung bildet die Basis für die Denkfigur der $\rightarrow$ erwartungswidrigen Schulleistung: Erzielen Schüler geringere Schulleistungen als man aufgrund ihres Intelligenzniveaus prognostizieren würde, werden sie als Underachiever (vgl. Hanses \& Rost, 1998; Sparfeldt et al., 2006), im umgekehrten Falle als Overachiever bezeichnet.

Die Fixierung auf allgemeine intellektuelle Fähigkeiten und inhaltsunabhängige Denkoperationen $(\rightarrow$ Intelligenz und Begabung) hat lange Zeit den Blick auf andere individuelle sowie instruktionale Determinanten der Schulleistung verstellt. Dies gilt auch für das aufgaben- und bereichsspezifische Vorwissen, das sich

\section{Konative Determinanten}

Zunehmend Interesse finden in der neueren pädagogisch-psychologischen Forschung konative (oder volitionale) Determinanten des Lernens und der Schulleistung (vgl. Snow et al., 1996). Darunter fallen $\rightarrow$ Lernstrategien und Lernstile, metakognitive Kompetenzen $(\rightarrow$ Metakognition), Lerngewohnheiten, Arbeitstechniken ( $\rightarrow$ Arbeitsverhalten und Arbeitsprobleme) und Handlungskontrolle, insbesondere die Fähigkeit zur Abschirmung bereits gebildeter Lernintentionen gegenüber konkurrierenden Tendenzen ( $\rightarrow$ Anstrengungsvermeidung; $\rightarrow$ Aufmerksamkeit und Konzentration). Die wenigen empirischen Untersuchungen zum $\mathrm{Zu}$ sammenhang mit Schulleistungen zeichnen allerdings ein uneinheitliches Bild mit überwiegend schwachen Zusammenhängen. Dies dürfte einerseits daran liegen, dass bspw. Lernstrategien oft über Schülereinschätzungen zur Anwendungshäufigkeit erfasst wurden (Artelt, 2000). Werden hingegen Lernstrategien wie bei PISA 2009 aufgabennah im Sinne von Strategiewissen gemessen, indem Schülerangaben mit Expertenlösungen verglichen werden (OECD, 2010), so fallen die Zusammenhänge mit den Schülerleistungen deutlicher und konsistenter aus (Schiefele, 2005; Abt Gürber et al., 2011). Andererseits könnte es auch daran liegen, dass diese Variablen in erster Linie für $\rightarrow$ selbstgesteuertes Lernen und nicht so sehr für das stark fremdgesteuerte, vom Lehrer strukturierte und kontrollierte Lernen bedeutsam sind (vgl. Corno \& Snow, 1986; Weinert \& Helmke, 1995b; Artelt, 2000). Abgesehen davon kann man sicher keine einfachen, direkten und linearen 
Zusammenhänge zwischen volitionalen Merkmalen und Schulleistung erwarten, sondern komplexe Wechselwirkungen und vielfältige Kompensationsmöglichkeiten, z. B. von ineffizienten Lernstrategien durch vermehrte Anstrengung (vgl. Klauer \& Leutner, 2012).

\section{Motivationale Bedingungen}

Im Gegensatz zu allgemeinen Persönlichkeitseigenschaften (wie den Big Five, bei denen lediglich die Gewissenhaftigkeit die Schulleistungen beeinflusst, s. Hattie, 2009) spielen motivationale, affektive und emotionale Bedingungen eine beachtliche Rolle für schulische Leistungen (Schrader \& Helmke, 2015). Umfassendere Übersichten über motivationale Bedingungen des Lernens sind bei Snow et al. (1996) oder Helmke und Weinert (1997) zu finden; für vertiefende Hinweise $\mathrm{zu}$ emotionalen und motivationalen Bedingungen des Lernens siehe insbesondere Pekrun und Schiefele (1996), Schiefele und Pekrun (1996), Hascher (2005) sowie Hattie und Anderman (2013) $(\rightarrow$ Interesse; $\rightarrow$ Anstrengungsvermeidung; $\rightarrow$ Intrinsische und extrinsische Motivation; $\rightarrow$ Leistungsängstlichkeit). Wir beschränken uns im Folgenden auf die Befundlage, die auf Metaanalysen beruht, wie sie von Hattie (2009) berichtet werden.

Das Fähigkeitsselbstkonzept (weitgehend deckungsgleich mit subjektiver Kompetenz, leistungsbezogenem Selbstvertrauen, Erfolgserwartung; $\rightarrow$ Selbstkonzept) beeinflusst schulische Leistungen mittels verschiedener Mechanismen (Mediationsprozesse wie Handlungsinitiierung, Abwehr von Störreizen, Aufrechterhaltung der Ausdauer bei auftretenden Schwierigkeiten, Pufferfunktion gegenüber selbstwertrelevanten Worry-Kognitionen), was sich in Korrelationen zwischen Fähigkeitsselbstkonzept und Schulleistung niederschlägt, die im Effektstärkebereich zwischen $d=0,32$ und $d=0,41$ liegen (Hattie, 2009, 2012). Die Korrelationen sind umso höher, je bereichsspezifischer das Fähigkeitsselbstbild konzeptualisiert wird (z. B. Rost et al., 2007; Schilling et al., 2005), je höher es ist und je älter die Schüler sind (vgl. Helmke, 1992). Allerdings sind die kausalen Beziehungen zwischen Selbstkonzept und Schulleistung komplex: In vielen Studien zeigen sich reziproke Muster der Beeinflussung: Selbstkonzept beeinflusst die Schulleistung (self-enhancement model), hängt aber auch von ihr ab (skill-development model; s. Helmke \& van Aken, 1995, und Valentine et al., 2004). Noch enger ist die Beziehung zwischen der Selbstwirk- samkeitsüberzeugung ( $\rightarrow$ Selbstwirksamkeit) und schulischen Leistungen (Multon et al., 1991; OECD, 2013a).

Lernmotivation, $\rightarrow$ Interesse und Einstellung zum Lernen (ähnlich: Lernfreude bzw. Lernunlust, Lerninvolvement) sind schulleistungsrelevante motivationale Konstrukte, die miteinander verwandt sind, aber verschiedene Facetten des affektiven Bezugs zum Lernen (oder generalisiert: zur Schule oder zu den Lehrpersonen) repräsentieren.

\section{Prüfungsangst und Leistungshandeln}

Prüfungsangst gehört zu den am häufigsten untersuchten Emotionen des Leistungshandelns (vgl. Cortina, 2008; Helmke, 1983; Hembree, 1988; $\rightarrow$ Leistungsängstlichkeit; $\rightarrow$ Emotionen im Lern- und Leistungskontext). Sie kann sich - in Abhängigkeit von der Bedrohlichkeit der Situation, der Aufgabenschwierigkeit und der subjektiven Kompetenz sowohl leistungsförderlich als auch leistungshemmend auswirken.

Man unterscheidet traditionell zwei Komponenten der Prüfungsangst: Emotionalität (Aufgeregtheit, Selbstwahrnehmung körperlicher Symptome; emotionality) und Besorgnis (Selbstzweifel; worry); als leistungsbeeinträchtigend hat sich vor allem die Besorgniskomponente herausgestellt. Prüfungsangst erschwert nicht nur den Abruf (retrieval) gelernter Information, sondern wirkt sich auch in der Vorbereitungs- und Lernphase ungünstig auf die Tiefe der Informationsverarbeitung und Informationsspeicherung (encoding) aus (Rost \& Schermer, 2007).

Unter Einstellung zum Lernen versteht man die affektiv (negativ oder positiv) getönte Orientierung gegenüber dem Lernen (Helmke, 1993) oder gegenüber bestimmten Fächern. $\rightarrow$ Interesse ist ein zentrales Element selbstbestimmten Handelns und Bestandteil intrinsischer Motivation (Schiefele et al., 1993; $\rightarrow$ Intrinsische und extrinsische Motivation). In Lernmotivation gehen sowohl subjektive Erwartungen als auch Anreizwerte ein (neben antizipierten Folgen wie Stolz, Trauer, Scham oder Reaktionen signifikanter anderer auch der Anreizwert der Lernhandlung selbst; »Tätigkeitsinteresse«, Rheinberg, 2004). Günstigenfalls ist der Anreizwert der Lernhandlung so ausgeprägt, dass es für die Initiierung und Aufrechterhaltung von Lernprozessen kei- 
ner zusätzlichen extrinsischer Motoren bedarf, z. B. im Falle des sog. Flowerlebens (Csikszentmihalyi, 1985; Csikszentmihalyi \& Schiefele, 1993).

Die Zusammenhänge zwischen der Schulleistung und diesen Konstrukten sind durchwegs positiv (Hattie, 2009), am stärksten beim Interesse mit einer durchschnittlichen Korrelation von $r=, 41$ (vgl. Schiefele \& Schreyer, 1994), schwächer bei den beiden anderen Konstrukten. Dies deutet darauf hin, dass der kausale Weg bis zur Schulleistung weit ist und Mediationsprozesse und Kontextvariablen ins Spiel kommen.

Ein weiteres relevantes Personenmerkmal ist die Selbstkontrolle. Langzeitstudien haben ergeben, dass Selbstkontrolle (self-control, self-discipline) im Kindesalter, meist erhoben durch die Fähigkeit zur Aufschuborientierung (delay of gratification) mit dem sog. Marshmallow-Test, einen erheblichen Teil der Streuung des Schul- und Berufserfolges, aber auch der Gesundheit im Jugend- und Erwachsenenalter aufklärt (Duckworth \& Seligman, 2005; Moffitt et al., 2011).

\section{Zusammenwirken verschiedener individueller Determinanten}

Die einzelnen Determinanten der Schulleistung weisen zum Teil nicht nur erhebliche konzeptuelle Überlappungen auf, sondern interagieren auch in komplexer Weise miteinander. Sie können z. B. im Verhältnis der Koppelung (für einen bestimmten Effekt ist das gleichzeitige Vorhandensein von Mindestausprägungen bei verschiedenen Variablen nötig) oder der Kompensation (ein und derselbe Effekt kann durch unterschiedliche hinreichende Bedingungen zustande kommen) zueinander stehen.

So setzen Höchstleistungen Mindestausprägungen der $\rightarrow$ Intelligenz und Begabung sowie der Anstrengung zwingend voraus (Koppelung), während bei Leistungen im Normalbereich mangelnde Begabung - solange diese nicht unter einen kritischen Schwellenwert fällt - durch vermehrte Anstrengungen kompensiert werden kann (und umgekehrt). In ähnlicher Weise können Prüfungsängstliche ihre angstbedingten Leistungsdefizite durch eine intensivere Vorbereitung und effizientere Lernstrategien wettmachen (Rost \& Schermer, 1991, 2007).

Kommunalitätenanalysen unterstreichen zwar die Dominanz kognitiver Lernvoraussetzungen, zeigen aber auch, dass ein substanzieller Anteil der Schulleis- tungsvarianz nur durch kognitive und motivationale Determinanten gemeinsam aufgeklärt werden kann; diese »Kommunalität« (oder »konfundierte Varianz«) kann als Kristallisierung der jahrelangen wechselseitigen Beeinflussung beider Variablengruppen interpretiert werden (Helmke, 1992).

\section{Familiäre Determinanten}

\section{Allgemeine familiäre Einflussfaktoren}

Genetische Einflüsse auf die Persönlichkeitsentwicklung und damit auch auf die Schulleistung werden nicht nur dadurch wirksam, dass die Eltern ihre Gene an ihre Kinder weitergeben, sondern auch aufgrund verschiedener Formen der Kovariation und Interaktion von Genotyp und Umwelt (Asendorpf, 1994; Weinert, 1994).

So schaffen intelligente Eltern meist auch eine der eigenen genetischen Prädisposition entsprechende - anregungsreiche, die Intelligenz der Kinder fördernde Umwelt. Da genetische Faktoren und Umwelteinflüsse konfundiert sind, ist eine eindeutige Schätzung der relativen Bedeutsamkeit beider Einflussfaktoren nicht möglich ( $\rightarrow$ Anlage und Umwelt). Es ist daher kurzschlüssig, Zusammenhänge zwischen Merkmalen des elterlichen $\rightarrow$ Erziehungsstils oder der familiären Lernumwelt mit der Schulleistung kausal als »Effekt « der Umwelt zu interpretieren; umgekehrt sind aber auch einseitig erbpsychologische Deutungen solcher Befunde unzulässig (vgl. Weinert, 1994; $\rightarrow$ Entwicklung, Lernen, Erziehung).

Die soziale und sprachliche Herkunft spielt im Bildungsbereich eine enorme Rolle, wie die internationalen Leistungsvergleichsstudien des letzten Jahrzehnts $(\rightarrow$ Internationale Schulleistungsvergleiche) belegen. Aus pädagogisch-psychologischer Sicht ist vor allem die Frage wichtig, in welchem Maße es dem Bildungssystem gelingt, den Zusammenhang zwischen sozialen Eingangsvoraussetzungen und Bildungserfolg $\mathrm{zu}$ entkoppeln. PISA zeigt für die untersuchten Fünfzehnjährigen, dass es erhebliche, wenn auch zwischen einzelnen Ländern deutlich differierende Zusammenhänge zwischen sozialer Herkunft und Leistung gibt (OECD, 2013b). Während Deutschland bei PISA 2000 noch zu den Ländern gehörte, bei denen der Zusammenhang zwischen sozialer Herkunft und Schülerleistung am höchsten war (Baumert \& Schümer, 2001), so waren 
bei PISA 2012 bezüglich des Zusammenhangs zwischen sozialer Herkunft (gemessen als ökonomische, soziale und kulturelle Ressourcen im Elternhaus) und Mathematikkompetenz keine signifikanten Unterschiede mehr zum OECD-Durchschnitt festzustellen (Müller \& Ehmke, 2013). Seit einigen Jahren lässt sich also in Deutschland ein positiver Trend feststellen, nämlich eine fortschreitende Entkoppelung zwischen sozialer Herkunft und Schülerleistungen, bei gleichzeitiger $\mathrm{Zu}$ nahme des Leistungsniveaus (OECD, 2013b).

In der im Jahr 2001 erstmals durchgeführten Grundschulstudie IGLU (Schwippert et al., 2003), in der Viertklässler untersucht wurden, ergaben sich in Deutschland anhand des auch bei PISA 2000 zugrunde gelegten Schichtindikators Unterschiede in der Leseleistung zwischen oberster und unterster Sozialschichtkategorie von ca. einer halben Standardabweichung, was einer Differenz von etwa einem Schuljahr entspricht. Der Zusammenhang zwischen sozialer Schicht und Leseleistung ist in der Grundschule (IGLU) somit deutlich geringer als bei der in PISA untersuchten Sekundarstufe. Ähnliche Zusammenhänge mit der sozialen Schicht lassen sich auch für die Leistungen in Mathematik und den Naturwissenschaften nachweisen, wobei alle drei Leistungsbereiche hoch miteinander korrelieren (zwischen $r=, 60$ und $r=, 74$ ). Dieser Befund bestätigte sich bei IGLU 2006 weitgehend (Bos et al., 2007). Es zeigt sich also für Deutschland, dass sich der bereits in der Grundschule nachweisbare deutliche Zusammenhang zwischen Leistung und sozialer Herkunft in der Sekundarstufe markant verstärkt.

Aufgrund der wiederholt gefundenen engen Zusammenhänge zwischen sozialer Herkunft und Schülerleistungen wurden Fragen der Chancengerechtigkeit neu diskutiert. Zurückgehend auf Breen und Goldthorpe (1997) werden primäre und sekundäre Bildungsdisparitäten unterschieden. Stoßend sind dabei weniger die primären Ungleichheiten, also der Befund, dass höhere Leistungen mit privilegierten familiären Verhältnissen einhergehen, sondern dass im Sinne sekundärer Ungleichheiten selbst bei gleichen fachlichen Kompetenzen der Besuch eines Gymnasiums stark von der sozialen Herkunft abhängt (Baumert \& Schümer, 2002; Ramseier \& Brühwiler, 2003).

Status- und Strukturvariablen wie Schichtzugehörigkeit, Familienkonstellation oder Berufstätigkeit der Mutter haben nur einen indirekten Erklärungswert für interindividuelle Unterschiede in der Schulleistung, denn die eigentlichen Wirkvariablen sind in schulleistungsrelevanten Merkmalen des Erziehungsstils oder der familiären Lernumwelt zu sehen ( $\rightarrow$ Erziehungsstil; $\rightarrow$ Eltern und Familie), bspw. in der Lesesozialisation (Stubbe et al., 2007).

\section{Prozessmerkmale des Elternverhaltens}

Beim überwiegenden Teil der Forschungsergebnisse zur Rolle des Elternverhaltens ( $\rightarrow$ Eltern und Familie), der Elternpersönlichkeit und der familiären Lernumwelt für die Schulleistung handelt es sich um Korrelationen auf der Basis von Querschnittsuntersuchungen, deren kausale Interpretation fragwürdig ist; experimentelle Untersuchungen, Trainings- oder Längsschnittstudien sind dagegen selten (vgl. Helmke \& Weinert, 1997).

So kann etwa eine mittelhohe Korrelation zwischen elterlichen Schulleistungserwartungen für das Kind und dessen Schulleistung bedeuten, dass die Schulleistung von den elterlichen Erwartungen beeinflusst wird, dass sich umgekehrt die Erwartungen der Eltern am Leistungsstand des Kindes orientieren, dass der Zusammenhang zwischen Erwartungen und Schulleistung durch Drittvariablen (genetische Variablen, Familienklima) vermittelt wird oder dass eine Kombination dieser Faktoren vorliegt.

Sichtet man die einschlägigen Längsschnittstudien, dann lassen sich schulleistungsrelevante Aspekte des Elternverhaltens wie folgt klassifizieren:

Stimulation. Dass eine anregende, das Kind aktivierende Umwelt, die viele Lerngelegenheiten bietet und das Neugiermotiv anspricht, bereits die sensumotorische Entwicklung und später die gesamte intellektuelle Entwicklung fördert, ist vielfach belegt worden (vgl. Trudewind \& Wegge, 1989). Die intellektuelle Stimulation in der familiären Lernumwelt ist ein stärkerer Prädiktor der Lernleistung als der sozioökonomische Status der Eltern, wie insbesondere die Metaanalyse von Gottfried (1984) mit der HOME-Skala (Home Observation for Measurement of the Environment) zeigte.

Instruktion. Eltern übernehmen häufig Aufgaben der direkten Förderung und der Instruktion (Sigel \& McGillicuddy-DeLisi, 1984; $\rightarrow$ Hausaufgaben; $\rightarrow$ Nachhilfeunterricht). Solche von den Eltern übernommenen Unterrichtsaufgaben können in kompensatorischem, konkurrierendem oder kooperativem Verhältnis zum schulischen Unterricht stehen (Davies \& Johnson, 1996). Helmke et al. (2004) fanden, dass Merkmale der elterlichen Unterstützung (Kontrolle, $\rightarrow$ Übung, 
Lernmanagement, Erklären; $\rightarrow$ Erziehungsstil) negativ mit der Leistung korrelieren, was dafür spricht, dass Eltern ihre Unterstützungsmaßnahmen eher kompensatorisch einsetzen. Die Forschung hat gezeigt, dass elterliche Instruktionsaktivitäten um so wirksamer sind, je besser die Passung mit den kognitiven und motivationalen Lernvoraussetzungen des Kindes ist, je stärker prozessorientiert sie sind (Hilfe zur Selbsthilfe, Strategieförderung statt bloßer Kontrolle oder direkter Unterstützung), je weniger direktiv sie erfolgen, je besser sie in ein positives, von Vertrauen, Akzeptanz und Verständnis bestimmtes Familienklima eingebettet sind $(\rightarrow$ Personzentrierte Unterrichtung und Erziehung) und je fachlich kompetenter die elterliche Hilfe erfolgt ( $\rightarrow$ Eltern und Familie). Dies wird auch durch die in der Hattie-Studie (2009) verwendeten Metaanalysen zur Rolle der Elternunterstützung beim Lernen bestätigt. Als besonders lernwirksam erwies es sich, wenn Eltern ihren Kindern spezifische Lese- und Schreibfertigkeiten beibringen.

Motivation. Eltern beeinflussen Lernverhalten und Leistungsergebnisse mittelbar dadurch, dass sie auf schulleistungsrelevante Motive, Einstellungen, Selbstkonzepte $(\rightarrow$ Selbstkonzept) und Orientierungen ihrer Kinder einwirken: durch ihre schulleistungs- und schulabschlussbezogenen Erwartungen, Aspirationen, Wünsche, Standards und Anspruchsniveaus, Sanktionen $(\rightarrow$ Paradoxe Effekte von Lob und Tadel; $\rightarrow$ Pädagogische Verhaltensmodifikation) und belief systems und schließlich durch Einschätzungen, Diagnosen und Prognosen der Kompetenzen ihrer Kinder $(\rightarrow$ Diagnostische Kompetenz von Eltern und Lehrern). So hat etwa Wild (2001) Zusammenhänge zwischen verschiedenen Aspekten elterlichen Schulengagements (Autonomieunterstützung, Strukturierung, motivationale Zuwendung, Stimulation; $\rightarrow$ Eltern und Familie) und der intrinsischen Lernmotivation gefunden, während Kontrolle mit extrinsischer Lernmotivation zusammenhängt $(\rightarrow$ Intrinsische und extrinsische Motivation).

Modellfunktion. Eltern sind wirkungsvolle Vorbilder für ihre Kinder: Viele Aspekte der elterlichen Persönlichkeit, insbesondere auch ihre Strategien des Umgangs mit Leistungsanforderungen, Erfolgen und Misserfolgen, werden von den Kindern durch Beobachtungslernen ( $\rightarrow$ Modelllernen) übernommen und beeinflussen dann mittelbar auch deren Schulleistung.

Oft wird von »Eltern « gesprochen, obwohl in Wirklichkeit nur Mütter untersucht wurden. Durch den
Einbezug der Väter - aber auch anderer signifikanter Familienmitglieder - in Wirkungsanalysen der familiären Umwelt würde das Bild der familiären $\rightarrow$ Sozialisation facettenreicher werden ( $\rightarrow$ Eltern und Familie). Beispielsweise bekäme man Aufschluss über Konkordanzen oder Dissonanzen von Vätern und Müttern in schulleistungsrelevanten Elternverhaltensweisen und deren Effekt auf kindliches Lernen.

\section{Rolle der Lehrperson und des Unterrichts}

\section{Unterrichtsqualität, Unterrichtsprogramme}

Die umfangreiche Forschung zur Rolle des Unterrichts kann hier nur kursorisch und selektiv skizziert werden $(\rightarrow$ Unterrichtsqualität; $\rightarrow$ Lehr-Lern-Forschung; $\rightarrow$ Instruktionspsychologie; $\rightarrow$ Lehrstrategien; $\rightarrow$ LehrerSchüler-Interaktion; $\rightarrow$ Aptitude-Treatment-Interaktion; $\rightarrow$ Entdeckendes Lernen; $\rightarrow$ Kooperatives Lernen; $\rightarrow$ Situiertes Lernen). Ausführliche Übersichten finden sich bei Einsiedler (1997), Helmke (2017), Helmke und Weinert (1997), Shuell (1996) und Weinert (1996). Neuere Untersuchungen haben gezeigt, dass die Quantität des Unterrichtsangebots und die Qualität des Unterrichtens einen wichtigen Einfluss auf die Schulleistung haben ( $\rightarrow$ Unterrichtsqualität), wenngleich ihr Erklärungsbeitrag erheblich geringer ist als derjenige der kognitiven Eingangsvoraussetzungen $(\rightarrow$ Intelligen $z$ und Begabung).

Einen anderen Zugang stellen Lehr- und Unterrichtsprogramme wie das Modell des Zielerreichenden Lernens (mastery learning), das Konzept des adaptiven Unterrichts oder aus der kognitiven Psychologie stammende Konzepte wie reziproke Instruktion (reciprocal teaching), kognitive Meisterlehre (cognitive apprenticeship), anchored instruction ( $\rightarrow$ Situiertes Lernen) oder conceptual change teaching dar (vgl. Shuell, 1996; $\rightarrow$ Lehrstrategien; $\rightarrow$ Instruktionspsychologie).

\section{Lehrermerkmale, Unterrichtsstile}

Statt mit isolierten Variablen der $\rightarrow$ Unterrichtsqualität beschäftigt sich die Unterrichtsstil-Forschung mit kohärenten Mustern des Unterrichtsverhaltens, die in der Regel mit bestimmten pädagogischen Orientierungen und Erziehungsphilosophien verknüpft sind (Calderhead, 1996; vgl. auch $\rightarrow$ Erziehungsstil; $\rightarrow$ Lehrer-SchülerInteraktion). Stilkonzepte wie z.B. der aus der Füh- 
rungsforschung abgeleitete lehrerzentrierte (autoritative, direkte) vs. schülerzentrierte (offene, demokratische; $\rightarrow$ Personzentrierte Unterrichtung und Erziehung) Unterrichtsstil haben sich jedoch als zu global und eindimensional herausgestellt, um die Forschung anzuregen oder für die Schulpraxis von Wert zu sein (zu ihren Effekten vgl. Helmke \& Weinert, 1997; Shuell, 1996).

Als fruchtbarer haben sich Forschungen zu verhaltenssteuernden intuitiven (subjektiven) Theorien, epistemologischen Überzeugungen sowie zu Merkmalen wie $\rightarrow$ Bezugsnormorientierung erwiesen. Einer der aussichtsreichsten Ansätze beschäftigt sich mit der $\rightarrow$ Expertise von Lehrern ( $\rightarrow$ Lehrerexpertise und Lehrerkompetenzen). Dabei wird versucht, Unterrichtsexperten oder »Meisterlehrer « zu identifizieren und durch Analyse ihrer Kompetenzen Aufschluss über die kognitiven Grundlagen des Lehrerhandelns zu gewinnen (vgl. Weinert et al., 1992).

Nachdem in den letzten beiden Dekaden die großen internationalen Vergleichsstudien $(\rightarrow$ Internationale Schulleistungsvergleiche) wie TIMSS (z.B. Beaton et al., 1996), PISA (z. B. OECD, 2013c) oder DESI (z. B. Klieme, 2008) vor allem Kompetenzmessungen bei den Schülern in den Blick nahmen, hat in den vergangenen Jahren das wissenschaftliche Interesse an der Lehrperson und an deren Bedeutung für Unterricht und Schulleistungen deutlich zugenommen (z.B. Richter et al., 2014). Zwar wurde in der Vergangenheit der Beitrag der Lehrperson für den akademischen Lernerfolg der Schüler oft kontrovers diskutiert. Beispielsweise korreliert nach Hattie (2009) das Fachwissen der Lehrpersonen nur äußerst schwach mit den Schülerleistungen $(d=0,09)$. Allerdings beruhen solche Befunde oft auf einer distalen Erfassung von fachlichem Wissen der Lehrpersonen (z. B. Qualifikationen oder Ausbildungsmerkmale). Wenn hingegen zur Messung des Lehrerwissens Leistungstests verwendet und in sog. value-added Studien die Leistungsentwicklung der Schüler längsschnittlich erfasst werden, fallen die Ergebnisse anders aus. So haben Hill et al. (2005) signifikante Effekte des mathematischen Wissens von Lehrpersonen auf die Entwicklung der Schülerleistungen während eines Schuljahrs gefunden. Auch Baumert und Kunter (2011a) kommen zum Schluss, dass ein hohes Fachwissen von Mathematiklehrpersonen notwendig ist, um vermittelt über fachdidaktisches Wissen kognitiv aktivierenden Unterricht halten zu können und so den
Lernerfolg der Schüler positiv zu beeinflussen. Heute gilt als empirisch gesichert, dass die Lehrperson und ihr unterrichtliches Handeln Schulleistungen maßgeblich beeinflussen (z. B. Lipowsky, 2006; Seidel \& Shavelson, 2007) und einen größeren Effekt auf die Schülerleistungen aufweisen als distale Faktoren wie Merkmale von Bildungssystemen oder Einzelschulen (z. B. Helmke $\&$ Weinert, 1997; Hattie, 2009; $\rightarrow$ Schuleffekte; $\rightarrow$ Schulqualität und Bildungsmonitoring).

Zur Beschreibung unterrichtsrelevanter Fähigkeiten von Lehrpersonen hat sich die Konzeption der professionellen Kompetenz von Lehrpersonen (z. B. Baumert $\&$ Kunter, 2011b) etabliert $(\rightarrow$ Lehrerexpertise und Lehrerkompetenzen). Das im Rahmen der COACTIVStudie ausgearbeitete Modell betrachtet nicht isolierte Teilfertigkeiten von Lehrpersonen, sondern ist multidimensional konzipiert und umfasst neben kognitiven Dispositionen (Professionswissen) auch motivationale und handlungsbezogene Aspekte wie Überzeugungen und Werthaltungen, motivationale Orientierungen und selbstregulative Fähigkeiten. Das Professionswissen als kognitive Dimension der professionellen Kompetenz besteht gemäß der allgemein akzeptierten Typologie nach Shulman $(1986,1987)$ und Bromme (1992) aus den drei Bereichen fachwissenschaftliches, fachdidaktisches sowie pädagogisch-psychologisches Wissen.

Neben den oben erwähnten Befunden zum fachlichen und fachdidaktischen Wissen mehren sich die empirischen Belege zur Bedeutung pädagogisch-psychologischen Wissens für das schulische Lernen. So konnten Voss et al. (2014) zeigen, dass das pädagogischpsychologische Wissen der Lehrperson eine wesentliche Voraussetzung für eine effiziente $\rightarrow$ Klassenführung und eine konstruktive Lernunterstützung darstellt. Auch eine hohe adaptive Lehrkompetenz (Beck et al., 2008; s. Kasten) erweist sich als günstige Voraussetzung für einen lernförderlichen Unterricht und höhere Leistungszuwächse bei den Schülern (Brühwiler, 2014).

\section{Leistungssteigerung bei möglichst allen Schülern} durch adaptive Lehrpersonen

Gelingt es Lehrpersonen, in ihren Klassen das Leistungsniveau möglichst aller Schüler, unabhängig vom Leistungsstand, von der sozialen Herkunft oder dem Sprachhintergrund, zu steigern? Aus zahlreichen Studien ist bekannt, dass Schüler mit niedrigem Leistungsniveau oder aus benachteiligten sozialen Verhältnissen von günstigen Lernbedingun- 
gen besonders profitieren (Scheerens \& Bosker, 1997). Solche differenziellen Effekte ( $\rightarrow$ AptitudeTreatment-Interaktion) sollten hingegen bei adaptiven Lehrpersonen (Beck et al., 2008), welche die individuellen Lernbedürfnisse der Schüler bei der Planung und Durchführung von Unterricht besonders berücksichtigen, nicht zu finden sein. Der Kern adaptiver Lehrkompetenz besteht aus einer engen Verzahnung von diagnostischer und didaktischer Kompetenz. Fachwissen und Klassenführungskompetenzen ( $\rightarrow$ Klassenführung) haben die Funktion, günstige Rahmenbedingungen $\mathrm{zu}$ schaffen. Es wurde untersucht, inwiefern adaptive Lehrkompetenz das unterrichtliche Handeln und die Schülerleistung beeinflusst (Brühwiler \& Blatchford, 2011; Brühwiler, 2014).

Stichprobe und Vorgehen. An der Studie beteiligten sich 49 Lehrpersonen und deren Klassen mit insgesamt 898 Schülern der Primarstufe (4./5. Schuljahr) und der Sekundarstufe I (7./8. Schuljahr). In einem Multi-Method-Ansatz wurde adaptive Lehrkompetenz mittels Vignettentest (Planungskompetenzen) und Videotest (Handlungskompetenzen) erfasst. Im Videotest mussten die Probanden anhand einer konstruierten Unterrichtssequenz kritische Situationen im Unterrichtsgeschehen erkennen und adaptive Handlungsalternativen formulieren. Die Erfassung der $\rightarrow$ Unterrichtsqualität erfolgte über einen Schülerfragebogen. Der Lernerfolg der Schüler (Leistungsfortschritt im Fach Biologie) wurde im Rahmen einer Unterrichtsreihe zum Thema »Keimung von Samen« gemessen. Zeitrahmen und Lernziele der Unterrichtsreihe waren einheitlich vorgegeben; wie die Lernziele vermittelt werden, war hingegen den Lehrpersonen überlassen. Auswertung. Zur Bestimmung der adaptiven Lehrkompetenz wurden die Lehrerantworten inhaltsanalytisch ausgewertet und quantifiziert. Mittels konfirmatorischer Faktorenanalysen konnte gezeigt werden, dass sich das theoretisch postulierte Konstrukt der adaptiven Lehrkompetenz empirisch identifizieren lässt. Um die hierarchische Datenstruktur (z.B. Lehrer- und Unterrichtsvariablen sowie Schülermerkmale) zu berücksichtigen, wurden mehrebenenanalytische Verfahren ( $\rightarrow$ Mehrebenenanalyse) eingesetzt.

Ergebnis. Eine hohe adaptive Lehrkompetenz wirkt sich positiv auf den Leistungszuwachs der Schüler während der Unterrichtsreihe aus - auch nach
Kontrolle möglicher alternativer Erklärungsgrößen (z. B. soziale Herkunft, Sprachhintergrund, Leistungsniveau der Klasse, Klassengröße). Innerhalb der Klassen profitieren alle Schülergruppen, unabhängig vom Vorwissensniveau, von der sozialen Herkunft oder vom Sprachhintergrund, ähnlich stark von adaptiven Lehrpersonen. Es lässt sich nachweisen, dass die Wirkung der adaptiven Lehrkompetenz auf die Schülerleistung über eine hohe $\rightarrow$ Unterrichtsqualität vermittelt wird, insbesondere über eine stärkere Individualisierung, eine bessere Vermittlungsqualität und eine höhere Regelorientierung.

Kommentar. Die empirischen Befunde sind angesichts der im Schulalltag vorzufindenden Vielfalt und Komplexität für die pädagogische Praxis von großer Bedeutung. Die Ausrichtung des Unterrichts an den unterschiedlichen Lernbedürfnissen der Schüler ist offenkundig auch in Regelklassen realisierbar und führt zu besseren Lernergebnissen. Aus methodischer Sicht scheint die kontextualisierte Erfassung von Lehrerkompetenzen (für eine Übersicht vgl. König, 2015) im Video- und Vignettentest aussichtsreich zu sein.

\section{Komplexe Unterrichtseffekte}

Einen Schritt weiter als die Untersuchung einfacher Unterrichtseffekte geht die Suche nach Wechselwirkungen zwischen Merkmalen des Unterrichts und der Schülerpersönlichkeit $(\rightarrow$ Aptitude-Treatment-Interaktion). Neuere Studien, die auf einem erweiterten Prozess-Produkt-Paradigma basieren, beschäftigen sich darüber hinaus insbesondere mit den folgenden vier Aspekten (vgl. Helmke, 1997, 2017):

(1) Multikriteriale Wirksamkeit. Schule und Unterricht verfolgen vielfältige Ziele im kognitiven, motivationalen, volitionalen und sozialen Bereich (Vermittlung von Kenntnissen und Fertigkeiten, Förderung von Kooperation, Fairness, selbstständigem Lernen, Emotionskontrolle, Selbstvertrauen). Gelegentlich harmonieren verschiedene Ziele nicht, sondern konkurrieren miteinander, sodass bestimmte Unterrichtsmethoden gegenläufige Effekte für verschiedene Zielkriterien haben. So zeigten Weinert und Helmke (1996), dass ein ausgeprägt aufgabenorientierter Unterricht mit intensiver Zeitnutzung zwar die Leistungen fördert, jedoch längerfristig $\mathrm{zu}$ einem nachhaltigen Abfall der Lernfreude 
führt. Dies macht deutlich, wie problematisch es angesichts multipler Zielkriterien wäre, global von "gutem" Unterricht zu sprechen (zur Konkurrenz von Zielkriterien vgl. etwa Helmke \& Schrader, 1990; Treinies \& Einsiedler, 1996; Kunter, 2005).

(2) Wechselseitige Kompensierbarkeit und Interaktion. Es gibt viele Möglichkeiten, "guten « und "schlechten« Unterricht $(\rightarrow$ Unterrichtsqualität) zu realisieren. So belegen die Ergebnisse der Münchner Studien (Weinert \& Helmke, 1987; vgl. auch Helmke, 1988a) enorme Unterschiede im unterrichtlichen Verhalten zwischen erfolgreichen Lehrern (gemessen an überdurchschnittlichen Zuwächsen in zentralen Zielkriterien). Dies deutet auf eine wechselseitige Kompensierbarkeit und Substituierbarkeit einzelner Qualitätsmerkmale des Unterrichts und von Kompetenzen von Lehrpersonen $(\rightarrow$ Lehrerexpertise und Lehrerkompetenzen) hin (Weinert \& Helmke, 1996).

$\mathrm{Zu}$ einem gewissen Ausmaß können folglich Defizite im einen Bereich durch besondere Stärken in anderen Bereichen kompensiert werden. So dürften sich Planungs- und Handlungskompetenzen von Lehrpersonen teilweise gegenseitig kompensieren lassen (Beck et al., 2008; Brühwiler, 2014). Wer schon bei der Planung unterschiedliche Unterrichtssituationen antizipiert und sich didaktische Maßnahmen überlegt, ist besser auf unvorhergesehene Unterrichtssituationen vorbereitet und muss geplanten Unterricht seltener bzw. weniger einschneidend anpassen.

Kompensatorische Effekte können entweder additiver (einzelne Merkmale können andere vollständig substituieren) oder multiplikativer Art (jeder Faktor muss eine Mindestausprägung aufweisen) sein und werden häufig in Form von Interaktionen beschrieben. So fanden Weinert und Helmke (1987), dass der Vorhersagewert des Fähigkeitsselbstbildes ( $\rightarrow$ Selbstkonzept) auf die Schulleistung vom Ausmaß der Unterstützung durch die Lehrperson abhängt: Je niedriger die Lehrerunterstützung, desto gewichtiger wird das Fähigkeitsselbstbild für die Schulleistung. Neuere Studien nehmen vermehrt auch Interaktionseffekte auf verschiedenen Ebenen in den Blick, etwa zwischen Unterrichtspraktiken und der Prozessqualität von Unterricht. So konnten Decristan et al. (2015) nachweisen, dass formative Beurteilung das naturwissenschaftliche Verständnis bei den Schülern stärker fördert, wenn Unterricht kognitiv aktivierend und in einem unterstützenden Lernklima stattfindet.
(3) Systemischer Charakter des Unterrichts und seiner Effekte. Von Unterricht als »Determinante« der Schulleistung zu sprechen, verführt zum Kurzschluss, es handele sich bei der Qualität des Unterrichts um eine »unabhängige Variable« in Form eines stabilen Merkmalsgefüges. In Wirklichkeit beeinflussen sich die Qualität des Unterrichts und Schulleistung (wie auch leistungsbezogene Orientierungen und Arbeitshaltungen) wechselseitig.

So beeinflussen Qualitätsmerkmale wie »Klarheit« und »Zeitnutzung» einerseits die spätere Mathematikleistung der Klasse positiv, hängen andererseits jedoch selbst vom anfänglichen Leistungsniveau der Klasse ab, wie insbesondere Ergebnisse der Münchner Hauptschulstudie zeigen: Je niedriger und unausgeglichener das Leistungsniveau der Klasse zum Zeitpunkt der Übernahme durch die Lehrkraft war, desto schwieriger fiel es ihr, »klaren « und »verständlichen« Unterricht zu realisieren, und desto mehr Zeit musste sie in disziplinarische, sozialpädagogische und remediale Aktivitäten - auf Kosten der Stoffbehandlung und damit des Leistungszuwachses - investieren (vgl. Helmke \& Weinert, 1997). Ähnliche Ergebnisse zur Rolle der Eingangsvoraussetzungen der Klasse für die $\rightarrow$ Unterrichtsqualität fanden sich in der Unterrichtsstudie DESI (Deutsch Englisch Schülerleistungen International) der KMK (Helmke et al., 2008). Aus theoretischer Sicht unterstreicht dies die multiple Determiniertheit der Schulleistung; aus anwendungsorientierter Sichtweise kann es als Warnung verstanden werden, die Erklärungslast für gute wie für schlechte Schulleistung vorschnell und einseitig einer einzigen Quelle - etwa schlechtem Unterricht - zuzuschlagen. Künftige Untersuchungen sollten dem systemischen Charakter des Zusammenspiels von Unterricht und Schulleistung dadurch Rechnung tragen, dass Unterrichtsqualität im Längsschnitt mehrfach erhoben wird (vgl. Helmke \& Renkl, 1993), sodass reziproke und bidirektionale Effekte modellierbar sind. (4) Kontextspezifität. Nur wenige Beziehungen zwischen instruktionalen, familiären und individuellen Variablen einerseits und der Schulleistung andererseits können für unterschiedliche Altersstufen, Aufgabentypen und Kontexte hinaus Gültigkeit beanspruchen. $\mathrm{Zu}$ den Aufgaben der Pädagogischen Psychologie gehört es, Modelle zu entwickeln und empirisch zu überprüfen, die so global wie möglich und so lokal und spezifisch wie nötig sein sollten (vgl. Snow \& Swanson, 1992). Die Perspektive, Merkmale des Unterrichts und 
der Schulklasse als Kontextvariable zu betrachten, die den Einfluss individueller Determinanten der Schulleistung moderiert, ist relativ neu, und die Ergebnisse solcher kontext- und klassenspezifischer Analysen stellen einige liebgewonnene Denkgewohnheiten (etwa die von der universellen, kontextunspezifischen Geltung von Prädiktor-Schulleistung-Zusammenhängen) ernsthaft in Frage.

So zeigte sich z. B. in den Münchner Studien, dass Leistungsangst ( $\rightarrow$ Leistungsängstlichkeit) keineswegs in allen untersuchten Schulklassen einen einheitlich leistungsbeeinträchtigenden Effekt hatte. Vielmehr variierte die Korrelation zwischen Leistungsangst und Schulleistung in der Grundschule von $r=-, 75$ bis $r=, 50$ (Helmke \& Weinert, 1999) und in der Hauptschule von $r=-, 81$ bis $r=, 36$ (Helmke, 1988b). In der Hauptschule erwies sich Angst besonders dann als leistungsmindernd, wenn die Zeitnutzung intensiv und der Unterricht eher unstrukturiert waren, während in der Grundschule Angst einen umso leistungsbeeinträchtigenderen Effekt hatte, je ungünstiger das sozio-emotionale Klima ( $\rightarrow$ Schul- und Klassenklima) und die affektive Beziehung zum Lehrer waren. Dieses Ergebnis unterstreicht neben der (Klassen-)Spezifität des Zusammenhangs zwischen Angst und Leistung zugleich die Altersspezifität, die sich durch die unterschiedliche Wertigkeit psychosozialer vs. kognitiver Ziele in beiden Altersbereichen ergibt. Das Bild wird noch komplizierter, wenn man Untersuchungen zur differenziellen Prädiktion von Schulleistung hinzu nimmt, die gezeigt haben, dass Schulleistungsprognosen $(\rightarrow$ Prognose von Schulerfolg) bei begabteren Schülern $(\rightarrow$ Hochbegabung) erfolgreicher sind (vgl. Heller, 1997).

Auch die Klassengröße kann als Kontextfaktor, der Effekte auf Unterrichtsprozesse und Schulleistungen moderiert, verstanden werden (Brühwiler \& Blatchford, 2011). So haben angelsächsische Studien nachgewiesen, dass kleine Klassen vor allem bei jüngeren Kindern einen positiven Effekt auf die individuelle Leistungsentwicklung ausüben (Finn \& Achilles, 1999; Blatchford et al., 2009). Leistungsschwächere und Kinder aus ethnischen Minderheiten sowie aus sozial benachteiligten Familien profitieren dabei besonders von kleineren Lerngruppen (Finn \& Achilles, 1999; Blatchford et al., 2003). In der britischen Längsschnittstudie CSPAR (Class Size and Pupil Adult Ratio) wurden Hinweise auf positive Auswirkungen von kleinen Klassen auf die $\rightarrow$ Unterrichtsqualität gefunden. In kleineren Klassen waren häufiger individualisierende und aufgabenbezogene $\rightarrow$ Lehrer-Schüler-Interaktionen, höhere Lernunterstützung sowie mehr Aufmerksamkeit für die einzelnen Schüler festzustellen als in größeren Klassen (Blatchford et al., 2007). Zudem kennen Lehrpersonen in kleinen Klassen die individuellen Lernvoraussetzungen besser (Brühwiler \& Blatchford, 2011; $\rightarrow$ Diagnostische Kompetenz von Eltern und Lehrern). Höhere Schulleistungen sind aber nicht einfach von der Klassengröße abhängig, sondern auch davon, ob das Potenzial kleinerer Klassen auch wirklich genutzt wird, etwa durch eine stärkere Individualisierung des Unterrichts (Blatchford et al., 2007; Brühwiler \& Blatchford, 2011); der von Hattie (2009) berichtete insgesamt nur schwach positive Effekt der Klassengröße auf den Lernerfolg $(d=0,23)$ deutet darauf hin, dass dies häufig nicht der Fall ist.

\section{Ausblick}

Die Suche nach den Determinanten der Schulleistung ist und bleibt eine Schlüsselfrage der $\rightarrow$ Lehr-Lern-Forschung, auch wenn sich die vorherrschenden Paradigmen und verwendeten Methoden im Laufe der Zeit geändert haben. Viele der dabei gewonnenen Erkenntnisse und entwickelten Methoden lassen sich mit Gewinn auch auf die Aufhellung des Determinationsgefüges der Leistungen in anderen Bereichen übertragen, z. B. in der Universität ( $\rightarrow$ Hochschuldidaktik) und der Weiterbildung.

Folgende Punkte erscheinen uns für die künftige Forschung zu den Determinanten der Schulleistung besonders aussichtsreich zu sein. Der Pseudo-Gegensatz zwischen »quantitativer « oder »qualitativer « Forschung sollte zugunsten einer Verknüpfung beider Forschungstypen überwunden werden (vgl. mixed methods; GläserZikuda et al., 2012). Intensive qualitative Analysen, z. B. Interviews mit Lehrern, Eltern und Schülern zu ihren intuitiven Konzepten von »Lehren « und »Lernen«, wären eine wichtige Ergänzung zu den vorherrschenden quantitativen Befunden (Helsper \& Klieme, 2013; Krüger \& Pfaff, 2004).

Angesichts der Dominanz von Large-scale-Studien $(\rightarrow$ Internationale Schulleistungsvergleiche) und korrelativen Auswertungsstrategien, die in der Regel keine klaren kausalen Schlüsse erlauben, wäre es wichtig, zunehmend auch experimentelle Ansätze, Trainings- 
studien und Interventionsstudien einzusetzen (Wellenreuther, 2005, 2009) sowie Ergebnisse aus der Evaluation von Schulentwicklungsprojekten zu berücksichtigen ( $\rightarrow$ Schulqualität und Bildungsmonitoring), sofern diese ein experimentelles oder zumindest quasiexperimentelles Design aufweisen. In diesem Zusammenhang sei auf das 2015 gestartete Längsschnittprojekt ALLE der Erziehungsdirektion Zürich hingewiesen, bei dem ein Bündel von lernförderlichen Maßnahmen (wie Aufgabenhilfe, Einsatz von Schulassistenzen, fachdidaktische Beratungsangebote und Weiterbildung bei der Diagnostik von Lehr-Lern-Prozessen) in ihrer Wirksamkeit auf den Lernverlauf empirisch geprüft wird (Volksschulamt Zürich, 2015).

Für vielversprechend halten wir auch kulturvergleichende Studien (Portes, 1996; Trommsdorff, 2007) zu den institutionellen, unterrichtlichen, familiären und individuellen Determinanten der Schulleistung. Die Vergleichsstudien ( $\rightarrow$ Internationale Schulleistungsvergleiche) der International Association for the Evaluation of Educational Achievement (IEA) und der OECD haben gezeigt, welch wichtige Rolle gesellschaftlichen und wirtschaftlichen Rahmenbedingungen zukommt (Stanat \& Lüdtke, 2007), wobei nicht zuletzt Vergleiche zwischen westlichen Industrieländern und Schwellenund Entwicklungsländern mit kontrastierender Schulorganisation, anderen Erziehungs- und Unterrichtszielen und abweichender sozialer Orientierung von Interesse sind (Helmke \& Schrader, 1999; Helmke et al., 2003; Rindermann et al., 2013), aber auch Vergleiche zwischen verschiedenen Kulturen innerhalb einer Gesellschaft. Ein Beispiel dafür ist der überragende Bildungserfolg von Schülern aus vietnamesischen Familien in Deutschland, trotz ungünstiger sozioökonomischer und sprachlicher Rahmenbedingungen innerhalb der Familie (Nauck \& Schnoor, 2015); ähnliche Ergebnisse wurden in den USA und Kanada für die Asian Americans gefunden (Zhou \& Lee, 2014). Dies sind Belege für die Rolle kultureller Faktoren, insbesondere des konfuzianischen Erbes, mit seiner hohen Wertschätzung von Leistung und Bildung, aber auch von Respekt vor Eltern und Lehrern.

\section{Literatur}

Einführende Literatur

Helmke, A. \& Weinert, F.E. (1997). Bedingungsfaktoren schulischer Leistungen. In F.E. Weinert
(Hrsg.), Psychologie des Unterrichts und der Schule (S. 71-176). Göttingen: Hogrefe.

Hasselhorn, M. \& Gold, A. (2013). Pädagogische Psychologie. Erfolgreiches Lernen und Lehren (3. Aufl.). Stuttgart: Kohlhammer.

Hattie, J.A.C. \& Anderman, E.M. (2013). International guide to student achievement. New York, NY: Routledge.

\section{Weiterführende Literatur}

Ackerman, P.L. \& Lohman, D.F. (2006). Individual differences in cognitive function. In P.A. Alexander \& P.H. Winne (Eds.), Handbook of educational psychology (2nd ed., pp. 139-161). Mahwah, NJ: Erlbaum.

Gustafsson, J.-E. \& Undheim, J.O. (1996). Individual differences in cognitive functions. In D.C. Berliner \& R.C. Calfee (Eds.), Handbook of educational psychology (pp. 186-242). New York, NY: Simon \& Schuster Macmillan.

Schrader, F-W. \& Helmke, A. (2015). School achievement: Motivational determinants and processes. In J.D. Wright (Ed.), International encyclopedia of the social \& behavioral sciences (2nd ed., Vol 21, pp. 48-54). Oxford: Elsevier. Snow, R., Corno, L. \& Jackson, D. (1996). Individual differences in affective and conative functions. In D.C. Berliner \& R.C. Calfee (Eds.), Handbook of educational psychology (pp. 243-310). New York, NY: Simon \& Schuster Macmillan.

\section{Zitierte Literatur}

Abt Gürber, N., Buccheri, G. \& Brühwiler, C. (2011). Engagement im Lesen und Lernstrategien. In Konsortium PISA.ch (Hrsg.), PISA 2009: Regionale und kantonale Ergebnisse, (S. 25-36). Bern: BBT/EDK und Konsortium PISA.ch.

Artelt, C. (2000). Strategisches Lernen. Münster: Waxmann. Asendorpf, J. (1994). Entwicklungsgenetik der Persönlichkeit. In K. Schneewind (Hrsg.), Psychologie der Erziehung und der Sozialisation (S. 107-134). Göttingen: Hogrefe.

Baumert, J. \& Kunter, M. (2011a). Das mathematikspezifische Wissen von Lehrkräften, kognitive Aktivierung im Unterricht und Lernfortschritte von Schülerinnen und Schülern. In M. Kunter, J. Baumert, W. Blum, U. Klusmann, S. Krauss \& M. Neubrand (Hrsg.), Professionelle Kompetenz von Lehrkräften. Ergebnisse des Forschungsprogramms COACTIV, (S. 163-192). Münster: Waxmann.

Baumert, J. \& Kunter, M. (2011b). Das Kompetenzmodell von COACTIV. In M. Kunter, J. Baumert, W. Blum, U. Klusmann, 
S. Krauss \& M. Neubrand (Hrsg.), Professionelle Kompetenz von Lehrkräften (S. 29-53). Münster: Waxmann.

Baumert, J. \& Schümer, G. (2001). Familiäre Lebensverhältnisse, Bildungsbeteiligung und Kompetenzerwerb. In Deutsches PISAKonsortium (Hrsg.), PISA 2000. Basiskompetenzen von Schülerinnen und Schülern im internationalen Vergleich (S. 323-407). Opladen: Leske + Budrich.

Baumert, J. \& Schümer, G. (2002). Familiäre Lebensverhältnisse, Bildungsbeteiligung und Kompetenzerwerb im nationalen Vergleich. In Deutsches PISA-Konsortium (Hrsg.), PISA 2000 - Die Länder der Bundesrepublik Deutschland im Vergleich, (S. 159-202). Opladen: Leske + Budrich.

Beaton, A.E., Martin, M.O., Mullis, I.V.S., Gonzalez, E.J., Smith, T.A. \& Kelly, D.L. (1996). Science achievement in the middle school years. IEA's Third International Mathematics and Science Studie. Chestnut, MA: TIMSS International Study Center, Boston College.

Beck, E., Baer, M., Guldimann, T., Bischoff, S., Brühwiler, C., Müller, P. et al. (2008). Adaptive Lehrkompetenz. Analyse und Struktur, Veränderbarkeit und Wirkung handlungssteuernden Lehrerwissens. Münster: Waxmann.

Blatchford, P., Bassett, P., Goldstein, H. \& Martin, C. (2003). Are class size differences related to pupils' educational progress and classroom processes? Findings from the Institute of Education Class Size Study of Children Aged 5-7 Years. British Educational Research Journal, 29, 709-730.

Blatchford, P., Russell, A., Bassett, P., Brown, P. \& Martin, C. (2007) The effect of class size on the teaching of pupils aged 7-11 years. School Effectiveness and School Improvement, 18, 147-172.

Blatchford, P., Russell, A. \& Brown, P. (2009). Teaching in large and small classes. In L.J. Saha \& A.G. Dworkin (Eds.), International handbook of research on teachers and teaching (pp. 779-790). New York, NY: Springer.

Bloom, B.S. (1976). Human characteristics and school learning. New York, NY: McGraw-Hill.

Bos, W., Hornberg S., Arnold, K.-H., Faust, G., Fried, L., Lankes, E.M. et al. (Hrsg.). (2007). IGLU 2006. Lesekompetenzen von Grundschulkindern in Deutschland im internationalen Vergleich. Münster: Waxmann.

Breen, R. \& Goldthorpe, J.H. (1997). Explaining educational differentials: Towards a formal rational action theory. Rationality and Society, 9, 275-305.

Bromme, R. (1992). Der Lehrer als Experte. Zur Psychologie des professionellen Wissens. Bern: Huber.

Brühwiler, C. (2014). Adaptive Lehrkompetenz und schulisches Lernen. Effekte handlungssteuernder Kognitionen von Lehrpersonen auf Unterrichtsprozesse und Lernergebnisse der Schülerinnen und Schüler. Münster: Waxmann.

Brühwiler, C. \& Blatchford, P. (2011). Effects of class size and adaptive teaching competency on classroom processes and academic outcome. Learning and Instruction, 21, 95-108.

Calderhead, J. (1996). Teachers: beliefs and knowledge. In D.C. Berliner \& R.C. Calfee (Eds.), Handbook of educational psychology (pp. 709-725). New York, NY: Simon \& Schuster Macmillan.
Carroll, J.B. (1963). A model of school learning. Teachers College Record, 64, 723-733.

Corno, L. \& Snow, R.E. (1986). Adapting teaching to individual differences among learners. In M.C. Wittrock (Ed.), Handbook of research on teaching (pp. 255-296). New York, NY: Macmillan.

Cortina, K.S. (2008). Leistungsängstlichkeit. In W. Schneider \& M. Hasselhorn (Hrsg.), Handbuch der Pädagogischen Psychologie (S. 50-61). Göttingen: Hogrefe.

Csikszentmihalyi, M. (1985). Das Flow-Erlebnis. Stuttgart: Klett-Cotta. Csikszentmihalyi, M. \& Schiefele, U. (1993). Die Qualität des Erlebens und der Prozess des Lernens. Zeitschrift für Pädagogik, 39, 207-221.

Davies, D. \& Johnson, V. (1996). Crossing boundaries: Family, community, and school partnerships. International Journal of Educational Research, 25, 1-106.

Decristan, J., Klieme, E., Kunter, M., Hochweber, J., Büttner, G., Fauth, B. et al. (2015). Embedded formative assessment and classroom process quality: How do they interact in promoting students' science understanding? American Educational Research Journal, 52, 1133-1159.

Duckworth, A.L. \& Seligman, M.E.P. (2005). Self-discipline outdoes IQ in predicting academic performance of adolescents. American Psychologist Society, 16, 939-944.

Einsiedler, W. (1997). Unterrichtsqualität und Leistungsentwicklung. Literaturüberblick. In F.E. Weinert \& A. Helmke (Hrsg.), Entwicklung im Grundschulalter (S. 225-240). Weinheim: Beltz. Finn, J.D. \& Achilles, C.M. (1999). Tennessee's class size study: Findings, implication, misconceptions. Educational Evaluation and Policy Analysis, 21, 97-109.

Gläser-Zikuda, M., Seidel, T., Rohlfs, C., Gröschner, A. \& Ziegelbauer, S. (Hrsg.). (2012). Mixed Methods in der empirischen Bildungsforschung. Münster: Waxmann.

Gottfried, A.W. (1984). Home environment and early cognitive development: Longitudinal research. Orlando, FL: Academic Press.

Gustafsson, J.-E. \& Undheim, J.O. (1996). Individual differences in cognitive functions. In D.C. Berliner \& R.C. Calfee (Eds.), Handbook of educational psychology (pp. 186-242). New York, NY: Simon \& Schuster Macmillan.

Hanses, P. \& Rost, D.H. (1998). Das »Drama« der hochbegabten Underachiever. Zeitschrift für Pädagogische Psychologie, 12, 53-71.

Harnischfeger, A. \& Wiley, D.E. (1976). The teaching-learning process in elementary schools: A synoptic view. Curriculum Inquiry, 6, 5-43.

Hascher, T. (2005). Emotionen im Schulalltag: Wirkungen und Regulationsformen. Zeitschrift für Pädagogik, 51, 610-625.

Hattie, J.A.C. (2009). Visible Learning. A synthesis of over 800 meta-analyses relating to achievement. London: Routledge.

Hattie, J.A.C. (2012). Visible Learning for Teachers. Maximizing impact on Learning. London: Routledge.

Hattie, J.A.C. \& E.M. Anderman (2013). Introduction. International guide to student achievement. New York, NY: Routledge. 
Heller, K. (1997). Individuelle Faktoren der Schulleistung. Literaturüberblick. In F.E. Weinert \& A. Helmke (Hrsg.), Entwicklung im Grundschulalter (S. 183-201). Weinheim: Beltz.

Helmke, A. (1983). Schulische Leistungsangst - Erscheinungsformen und Entstehungsbedingungen. Frankfurt: Lang. tungsunterschieden in Schulklassen: Unvereinbare Ziele? Zeitschrift für Entwicklungspsychologie und Pädagogische Psychologie, 10, 45-76.

Helmke, A. (1988b). The role of classroom context factors for the achievement-impairing effect of test anxiety. Anxiety Research, 1, 37-52.

Helmke, A. (1992). Selbstvertrauen und schulische Leistungen. Göttingen: Hogrefe.

Helmke, A. (1993). Die Entwicklung der Lernfreude vom Kindergarten bis zur 5. Klassenstufe. Zeitschrift für Pädagogische Psychologie, 7, 77-86.

Helmke, A. (1997). Educational research on classroom instruction and its effects: Shortcomings, dead ends, and future perspective. In K.H. Rebel \& W. Bünder (Eds.), Teacher education - Theoretical requirements and professional quality (pp. 55-68). Kiel: IPN.

Helmke, A. (2017). Unterrichtsqualität und Lehrerprofessionalität. Diagnose, Evaluation und Verbesserung des Unterrichts (7. Aufl.). Seelze: Klett-Kallmeyer.

Helmke, A., Helmke, T., Schrader, F.-W., Wagner, W., Klieme, E., Nold, G. \& Schröder, K. (2008). Wirksamkeit des Englischunterrichts. In DESI-Konsortium (Hrsg.), Unterricht und Kompetenzerwerb in Deutsch und Englisch. Ergebnisse der DESIStudie (S. 382-397). Weinheim: Beltz.

Helmke, A. \& Renkl, A. (1993). Unaufmerksamkeit in Grundschulklassen: Problem der Klasse oder des Lehrers? Zeitschrift für Entwicklungspsychologie und Pädagogische Psychologie, 25, 185-205.

Helmke, A. \& Schrader, F.-W. (1990). Zur Kompatibilität kognitiver, affektiver und motivationaler Zielkriterien des Schulunterrichts - Clusteranalytische Studien. In M. Knopf \& W. Schneider (Hrsg.), Entwicklung (S. 180-200). Göttingen: Hogrefe.

Helmke, A. \& Schrader, F.-W. (1999). Lernt man in Asien anders? Empirische Untersuchungen zu studentischem Lernverhalten in Deutschland und Vietnam. Zeitschrift für Pädagogik, 45, 81-102.

Helmke, A., Schrader, F.-W. \& Hosenfeld, I. (2004). Elterliche Lernunterstützung und Schulleistungen ihrer Kinder. Bildung und Erziehung, 57, 251-277.

Helmke, A., Schrader, F.-W., Vo, T.A.T., Le, D.P. \& Tran, T.B.T. (2003). Selbstkonzept und schulische Leistungen im Kulturvergleich: Ergebnisse der Grundschulstudie SCHOLASTIK in München und Hanoi. In W. Schneider \& M. Knopf (Hrsg.), Entwicklung, Lehren und Lernen: Zum Gedenken an Franz Emanuel Weinert (S. 187-206). Göttingen: Hogrefe.

Helmke, A. \& van Aken, M. (1995). The causal ordering of academic achievement and self-concept of ability during elementary school: A longitudinal study. Journal of Educational Psychology, 87, 624-637.

Helmke, A. \& Weinert, F.E. (1997). Bedingungsfaktoren schulischer Leistungen. In F.E. Weinert (Hrsg.), Psychologie des Unterrichts und der Schule (S. 71-176). Göttingen: Hogrefe.

Helmke, A. \& Weinert, F.E. (1999). Schooling and the development of achievement differences. In F.E. Weinert \& W. Schneider (Eds.), Individual development from 3 to 12: Findings from the Munich longitudinal study (pp. 176-192). Cambridge: Cambridge University Press.

Helsper, W. \& Klieme, E. (2013). Quantitative und qualitative Unterrichtsforschung - Sondierung. Zeitschrift für Pädagogik, 59, 283-290.

Hembree, R. (1988). Correlates, causes, effects, and treatment of test anxiety. Review of Educational Research, 58, 47-77.

Hill, H.C., Rowan, B. \& Loewenberg Ball, D. (2005). Effects of teachers' mathematical knowledge for teaching on student achievement. American Educational Research Journal, 42, 371-406.

Klauer, K.J. \& Leutner, D. (2012). Lehren und Lernen. Einführung in die Instruktionspsychologie (2. Aufl.). Weinheim: Beltz.

Klieme, E. (Hrsg.). (2008). Unterricht und Kompetenzerwerb in Deutsch und Englisch. Ergebnisse der DESI-Studie. Weinheim: Beltz.

König, J. (2015). Kontextualisierte Erfassung von Lehrerkompetenzen. Einführung in den Thementeil. Zeitschrift für Pädagogik, 61, 305-309.

Krüger, H.-H. \& Pfaff, N. (2004). Triangulation quantitativer und qualitativer Zugänge in der Schulforschung. In W. Helsper \& J. Böhme (Hrsg.), Handbuch der Schulforschung (S. 159-182). Wiesbaden: VS Verlag für Sozialwissenschaften.

Kunter, M. (2005). Multiple Ziele im Mathematikunterricht. Münster: Waxmann.

Lipowsky, F. (2006). Auf den Lehrer kommt es an. Empirische Evidenzen für Zusammenhänge zwischen Lehrerkompetenzen, Lehrerhandeln und dem Lernen der Schüler. Zeitschrift für Pädagogik, 51 (Beiheft), 47-70.

Moffitt, T.E., Arseneault, L., Belsky, D., Dickson, N., Hancox, R.J., Harrington, H. et al. (2011). A gradient of childhood self-control predicts health, wealth, and public safety. Proceedings of the National Academy of Sciences, 108, 2693-2698.

Müller, K. \& Ehmke, T. (2013). Soziale Herkunft als Bedingung der Kompetenzentwicklung. In M. Prenzel, C. Sälzer, E. Klieme \& O. Köller (Hrsg.), PISA 2012. Fortschritte und Herausforderungen in Deutschland, (S. 245-274). Münster: Waxmann.

Multon, K.D., Brown, S.D. et al. (1991). Relation of self-efficacy beliefs to academic outcomes: A meta-analytic investigation. Journal of Counseling Psychology, 38, 30-38.

Nauck, B. \& Schnoor, B. (2015). Against all odds? Bildungserfolg in vietnamesischen und türkischen Familien in Deutschland. In Kölner Zeitschrift für Soziologie und Sozialpsychologie, 67, 633-657.

OECD (2010). PISA 2009 Results: Learning to learn. Student engagement, strategies and practices (Vol. III). Paris: OECD Publishing.

OECD (2013a). PISA 2012 Results: Ready to learn: Students' engagement, drive and self-beliefs (Vol. III). Paris: OECD Publishing.

OECD (2013b). PISA 2012 Results. Excellence through equity. Giving every student the chance to succeed (Vol. II). Paris: OECD Publishing. 
OECD (2013c). PISA 2012 Results. What students know and can do: Student performance in mathematics, reading and science (Vol. I). Paris: OECD Publishing.

Pekrun, R. \& Schiefele, U. (1996). Emotions- und motivationspsychologische Bedingungen der Lernleistung. In F.E. Weinert (Hrsg.), Psychologie des Lernens und der Instruktion (S. 154-180). Göttingen: Hogrefe.

Portes, P. (1996). Ethnicity and culture in educational psychology. In D.C. Berliner \& R.C. Calfee (Eds.), Handbook of educational psychology (pp. 331-357). New York, NY: Simon \& Schuster Macmillan.

Ramseier, E. \& Brühwiler, C. (2003). Herkunft, Leistung und Bildungschancen im gegliederten Bildungssystem: Vertiefte PISA-Analyse unter Einbezug der kognitiven Grundfähigkeiten. Schweizerische Zeitschrift für Bildungswissenschaften, 25, 23-58. Rheinberg, F. (2004). Motivation (5. Aufl.). Stuttgart: Kohlhammer. Richter, D., Stanat, P. \& Pant, H.A. (2014). Die Rolle der Lehrkraft für die Unterrichtsqualität und den Lernerfolg von Schülerinnen und Schülern. Zeitschrift für Pädagogik, 60, 181-183.

Rindermann, H., Hoang, Q.S.N. \& Baumeister, A.E.E. (2013). Cognitive ability, parenting and instruction in Vietnam and Germany. Intelligence, 41, 366-377.

Rost, D.H. (2013). Handbuch Intelligenz. Weinheim: Beltz.

Rost, D.H. \& Schermer, F.J. (1991). Dimensionen der Leistungsangstauslösung. Zeitschrift für Pädagogische Psychologie, 5, 131-144.

Rost, D.H. \& Schermer, F.J. (2007). DAI. Differentielles Leistungsangst Inventar (2. Aufl.). Frankfurt: Harcourt Test Services.

Rost, D.H., Sparfeldt, J.R. \& Schilling, S.R. (2007). DISK-Gitter mit SKSLF. Differentielles Schulisches Selbstkonzept-Gitter mit Skala zur Erfassung des Selbstkonzepts schulischer Leistungen und Fähigkeiten. Göttingen: Hogrefe.

Scheerens, J. \& Bosker, R.J. (1997). The foundations of educational effectiveness. Oxford: Elsevier.

Schiefele, U. (2005). Prüfungsnahe Erfassung von Lernstrategien und deren Vorhersagewert für nachfolgende Lernleistungen. In C. Artelt \& B. Moschner (Hrsg.), Lernstrategien und Metakognition: Implikationen für Forschung und Praxis (S. 13-41). Münster: Waxmann.

Schiefele, U., Krapp, A. \& Schreyer, I. (1993). Metaanalyse des Zusammenhangs von Interesse und schulischer Leistung. Zeitschrift für Experimentelle und Angewandte Psychologie, 25, 120-148.

Schiefele, U. \& Pekrun, R. (1996). Psychologische Modelle des fremdgesteuerten und selbstgesteuerten Lernens. In F.E. Weinert (Hrsg.), Psychologie des Lernens und der Instruktion (S. 249-278). Göttingen: Hogrefe.

Schiefele, U. \& Schreyer, I. (1994). Intrinsische Lernmotivation und Lernen. Zeitschrift für Pädagogische Psychologie, 8, 1-13.

Schilling, S.R., Sparfeldt, J.R., Rost, D.H. \& Nickels, G. (2005). Schulische Selbstkonzepte - Zur Validität einer erweiterten Version des Differentiellen Selbstkonzept-Gitters (DISK-Gitter). Diagnostica, 51, 21-28.

Schrader, F.-W. (2006). Kognitive Voraussetzungen. In K.-H. Arnold, U. Sandfuchs \& J. Wiechmann (Hrsg.), Handbuch Unterricht (S. 569-574). Bad Heilbrunn: Klinkhardt.
Schrader, F-W. \& Helmke, A. (2015). School achievement: Motivational determinants and processes. In J.D. Wright (Ed.), International encyclopedia of the social \& behavioral sciences (2nd ed., Vol. 21, pp. 48-54). Oxford: Elsevier.

Schwippert, K., Bos, W. \& Lankes, E.-M. (2003). Heterogenität und Chancengleichheit am Ende der vierten Jahrgangsstufe im internationalen Vergleich. In W. Bos, E.-M. Lankes, M. Prenzel, K. Schwippert, G. Walther \& R. Valtin (Hrsg.), Erste Ergebnisse aus IGLU (S. 265-302). Münster: Waxmann.

Seidel, T. \& Shavelson, R.J. (2007). Teaching effectiveness research in the last decade: The role of theory and research design in disentangling meta-analysis results. Review of Educational Research, 77, 454-499.

Shuell, T. (1996). Teaching and learning in the classroom context. In D.C. Berliner \& R.C. Calfee (Eds.), Handbook of educational psychology (pp. 726-764). New York, NY: Simon \& Schuster Macmillan.

Shulman, L.S. (1986). Paradigms and research programs in the study of teaching: A contemporary perspective. In M.C. Wittrock (Ed.), Handbook of Research on Teaching (pp. 3-36). New York, NY: MacMillan.

Shulman, L.S. (1987). Knowledge and teaching: Foundations of the new reform. Harvard Educational Review, 57, 1-22.

Sigel, I.E. \& McGillicuddy-DeLisi, A.V. (1984). Parents as teachers of their children: A distancing behavior model. In A.D. Pellegrino \& T.D. Yawkey (Eds.), The development of oral and written language in social contexts (pp. 71-92). Norwood, NJ: Ablex.

Snow, R., Corno, L. \& Jackson, D. (1996). Individual differences in affective and conative functions. In D.C. Berliner \& R.C. Calfee (Eds.), Handbook of educational psychology (pp. 243-310). New York, NY: Simon \& Schuster Macmillan.

Snow, R.E. \& Swanson, J. (1992). Instructional psychology: Aptitude, adaptation, and assessment. Annual Review of Psychology, 43, 583-626.

Sparfeldt, J.R., Schilling, S.R. \& Rost, D.H. (2006). Hochbegabte Underachiever als Jugendliche und junge Erwachsene. Des Dramas zweiter Akt? Zeitschrift für Pädagogische Psychologie, 20, 213-224.

Stanat, P. \& Lüdtke, O. (2007). Internationale Schulleistungsvergleiche. In G. Trommsdorff \& H.J. Kornadt (Hrsg.), Anwendungsfelder der kulturvergleichenden Psychologie (S. 279-347). Göttingen: Hogrefe.

Steiner, G. (1996). Lernverhalten, Lernleistung und Instruktionsmethoden. In F.E. Weinert (Hrsg.), Psychologie des Lernens und der Instruktion (S. 279-318). Göttingen: Hogrefe.

Stubbe, T.C., Buddeberg, I., Hornberg, S. \& McElvany, N. (2007). Lesesozialisation im Elternhaus im internationalen Vergleich. In W. Bos, S. Hornberg, K.-H. Arnold, G. Faust, L. Fried, E.-M. Lankes et al. (Hrsg.), IGLU 2006. Lesekompetenzen von Grundschulkindern in Deutschland im internationalen Vergleich (S. 299-327). Münster: Waxmann.

Treinies, G. \& Einsiedler, W. (1996). Zur Vereinbarkeit von Steigerung des Lernleistungsniveaus und Verringerung von Leistungsunterschieden in Grundschulklassen. Unterrichtswissenschaft, 24, 290-311. 
Trommsdorff, G. (2007). Entwicklung im kulturellen Kontext. In G. Trommsdorff \& H.J. Kornadt (Hrsg.), Erleben und Handeln im kulturellen Kontext (S. 435-519). Göttingen: Hogrefe.

Trudewind, C. \& Wegge, J. (1989). Anregung - Instruktion Kontrolle: Die verschiedenen Rollen der Eltern als Lehrer. Unterrichtswissenschaft, 17, 133-155.

Valentine, J.C., DuBois, D.L. \& Cooper, H. (2004). The relations between self-beliefs and academic achievement: A systematic review. Educational Psychologist, 39, 111-133.

Volksschulamt Zürich (2015). Aktive Lernzeit und Lernerfolg für ALLE. Verfügbar unter http://www.vsa.zh.ch/internet/bildungsdirektion/vsa/de/schulbetrieb_und_unterricht/projekte/pilotprojekt_alle.html [06.09.2015].

Voss, T., Kunter, M., Seiz, J., Hoehne, V. \& Baumert, J. (2014). Die Bedeutung des pädagogisch-psychologischen Wissens von angehenden Lehrkräften für die Unterrichtsqualität. Zeitschrift für Pädagogik, 60, 184-201.

Walberg, H.J. (1990). A theory of educational productivity: Fundamental substance and method. In P. Vedder (Ed.), Fundamental studies in educational research (pp. 19-34). Lisse: Swets \& Zeitlinger.

Wang, M.C., Haertel, G.D. \& Walberg, H.J. (1993). Toward a knowledge base for school learning. Review of Educational Research, 63, 249-294.

Weinert, F.E. (1994). Entwicklung und Sozialisation der Intelligenz, der Kreativität und des Wissens. In K.A. Schneewind (Hrsg.), Psychologie der Erziehung und Sozialisation (S. 259-284). Göttingen: Hogrefe.
Weinert, F.E. (1996). Lerntheorien und Instruktionsmodelle. In F.E. Weinert (Hrsg.), Psychologie des Lernens und der Instruktion (S. 1-48). Göttingen: Hogrefe.

Weinert, F.E. \& Helmke, A. (1987). Schulleistungen - Leistungen der Schule oder der Kinder? Bild der Wissenschaft, 24, 62-73.

Weinert, F.E. \& Helmke, A. (1995a). Inter-classroom differences in instructional quality and interindividual differences in cognitive development. Educational Psychologist, 30, 15-20.

Weinert, F.E. \& Helmke, A. (1995b). Learning from wise mother nature or big brother instruction: The wrong alternative for cognitive development. Educational Psychologist, 30, 135-142.

Weinert, F.E. \& Helmke, A. (1996). Der gute Lehrer: Person, Funktion oder Fiktion? In A. Leschinsky (Hrsg.), Die Institutionalisierung von Lehren und Lernen (S. 223-233). Weinheim: Beltz.

Weinert, F.E., Helmke, A. \& Schrader, F. W. (1992). Research on the model teacher and the teaching model: Theoretical contradiction or conglutination? In F. Oser, A. Dick \& J.L. Patry (Eds.), Effective and responsible teaching: The new synthesis (pp. 249-260). New York, NY: Jossey-Bass.

Wellenreuther, M. (2005). Lehren und Lernen - aber wie? Empirisch-experimentelle Forschungen zum Lehren und Lernen im Unterricht (2. Aufl.). Baltmannsweiler: Schneider.

Wellenreuther, M. (2009). Forschungsbasierte Schulpädagogik. Anleitungen zur Nutzung empirischer Forschung für die Schulpraxis. Baltmannsweiler: Schneider.

Wild, E. (2001). Familiale und schulische Bedingungen der Lernmotivation. Zeitschrift für Pädagogik, 47, 481-500.

Zhou, M. \& Lee, J. (2014). Assessing what is cultural about Asian Americans' academic advantage. Proceedings of the National Academy of Sciences of the United States of America, 111, 8321-8322.

\section{Diagnostische Kompetenz von Eltern und Lehrern}

Friedrich-Wilhelm Schrader • Anna-Katharina Praetorius

\section{Begriffsklärung und Bedeutung}

Mit Diagnostischer Kompetenz (engl. diagnostic competence, judgment accuracy) von Eltern und Lehrern bezeichnet man deren Fähigkeit, die bei der Ausübung ihrer Rolle anfallenden Diagnoseleistungen so zu erbringen, dass sie bestimmten Güte- oder Qualitätskriterien genügen. Diagnostische Aktivitäten beziehen sich auf die Gewinnung und Verarbeitung von Informationen, die für die Beurteilung von Personen wichtig sind. Sie können auf explizite, systematisch und reflektiert gewonnene sowie methodisch abgesicherte Aussagen (formelle Diagnostik) abzielen oder in subjektiven Ein- schätzungen, die oft implizit bleiben (informelle Diagnostik), resultieren. Formelle Diagnosen, einschließlich der zu ihrer Gewinnung verwendeten Methoden (z.B. $\rightarrow$ Schultests), sind Gegenstand der $\rightarrow$ pädagogisch-psychologischen Diagnostik. Bei informellen Diagnosen handelt es sich dagegen um Urteile, die nicht durch formelle Diagnosen abgestützt sind. Im pädagogischen Alltag, in dem eine methodisch fundierte formelle Diagnostik aus verschiedenen Gründen (z.B. zeitliche Restriktionen) oft nicht möglich ist, spielt die informelle Diagnostik eine wichtige Rolle für die Optimierung vieler Entscheidungen und Maßnahmen. Die Qualität dieser informellen Urteilsleistungen bildet den 